\title{
Novel mutations of PKD genes in Chinese patients suffering from autosomal dominant polycystic kidney disease and seeking assisted reproduction
}

\author{
Wen-Bin $\mathrm{He}^{1,2 \dagger}$, Wen-Juan Xiao ${ }^{1 \dagger}$, Yue-Qiu Tan ${ }^{1,2}$, Xiao-Meng Zhao ${ }^{2}$, Wen $\mathrm{Li}^{1,2}$, Qian-Jun Zhang ${ }^{1,2}$,
}

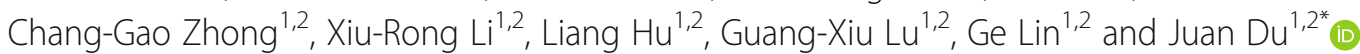

\begin{abstract}
Background: Autosomal dominant polycystic kidney disease (ADPKD), the commonest inherited kidney disease, is generally caused by heterozygous mutations in PKD1, PKD2, or GANAB (PKD3).

Methods: We performed mutational analyses of PKD genes to identify causative mutations. A set of 90 unrelated families with ADPKD were subjected to mutational analyses of PKD genes. Genes were analysed using long-range $P C R$ (LR-PCR), direct PCR sequencing, followed by multiplex ligation-dependent probe amplification (MLPA) or screening of GANAB for some patients. Semen quality was assessed for 46 male patients, and the correlation between mutations and male infertility was analysed.

Results: A total of 76 mutations, including 38 novel mutations, were identified in 77 families, comprising 72 mutations in PKD1 and 4 in PKD2, with a positive detection rate of $85.6 \%$. No pathogenic mutations of GANAB were detected. Thirty-seven patients had low semen quality and were likely to be infertile. No association was detected between PKD1 mutation type and semen quality. However, male patients carrying a pathogenic mutation in the Ig-like repeat domain of PKD1 had a high risk of infertility.

Conclusion: Our study identified a group of novel mutations in PKD genes, which enrich the PKD mutation spectrum and might help clinicians to make precise diagnoses, thereby allowing better family planning and genetic counselling. Men with ADPKD accompanied by infertility should consider intracytoplasmic sperm injection combined with preimplantation genetic diagnosis to achieve paternity and obtain healthy progeny.
\end{abstract}

Keywords: Autosomal dominant polycystic kidney disease, PKD1 gene, PKD2 gene, GANAB gene, Novel mutation, Male infertility

\section{Background}

Autosomal dominant polycystic kidney disease (ADPKD) is the commonest inherited kidney disease, with an estimated incidence of 1:400 to 1:1000; it accounts for 7$10 \%$ of all patients on renal replacement therapy worldwide [1]. It is characterised by the development of renal

\footnotetext{
* Correspondence: tandujuan@csu.edu.cn

${ }^{+}$Wen-Bin He and Wen-Juan Xiao contributed equally to this work. 'Institute of Reproductive and Stem Cell Engineering, School of Basic Medical Science, Central South University, Changsha, Hunan 410078, People's Republic of China

${ }^{2}$ Reproductive and Genetic Hospital of CITIC-Xiangya, Changsha, Hunan 410078, People's Republic of China
}

cysts, hypertension, and extrarenal cysts, and results in end-stage renal disease (ESRD) [2]. In addition, male patients with ADPKD usually show infertility resulting from cystic dilatation of the seminal vesicles [3].

ADPKD is an autosomal dominant inherited disorder resulting from heterozygous mutations in three genes: $P K D 1, P K D 2$, and GANAB. Mutations of the first two genes (PKD1 and PKD2) account for $80-85 \%$ and $15-$ $20 \%$ of resolved cases, respectively $[4,5]$. As of January 31, 2018, more than 2000 mutations (2323 in PKD1 and 278 in PKD2) had been described in the Autosomal Dominant Polycystic Kidney Disease Mutation Database 
(PKDB; http://pkdb.mayo.edu/). Recently, two studies reported the association of the third ADPKD gene GANAB, or PKD3 [6, 7], which accounts for $\sim 0.3 \%$ of the total cases of ADPKD [7].

In the present study, we performed mutational screening of PKD1, PKD2, and GANAB using long-range PCR (LR-PCR) and direct sequencing, as well as multiplex ligation-dependent probe amplification (MLPA) in 90 unrelated Chinese families with ADPKD. A total of 76 likely pathogenic or pathogenic mutations were identified in 77 families, including 38 novel mutations in PKD genes. These mutation data will contribute to improvement of diagnostics and genetic counselling in a clinical setting. In addition, this study highlights the correlation between men with ADPKD and infertility.

\section{Methods}

\section{Study subjects}

A total of 90 unrelated families were recruited from the Reproductive and Genetic Hospital of CITIC-Xiangya in China from October 2012 to October 2017, including 72 patients with a positive family history of ADPKD. These patients either sought genetic counselling to avoid delivering a baby with ADPKD due to a positive family history, or sought treatment at our hospital for infertility and were diagnosed with ADPKD based on ultrasound examination before undergoing assisted reproductive technology treatment. All diagnoses were confirmed by ultrasound examination according to previously described criteria: (1) the presence of at least three (unilateral or bilateral) renal cysts in individuals aged 15 to 39 years, or (2) the presence of at least two cysts in each kidney in individuals aged 40 to 59 years, or (3) the presence of four or more cysts in each kidney in individuals aged 60 years and above [8].

All individuals signed a written informed consent form, and blood samples were obtained from all probands and their family members when possible. The study was approved by the Ethics Committee of the Reproductive and Genetic Hospital of CITIC-Xiangya.

\section{Semen analysis and assisted reproductive therapies}

Among the 90 unrelated ADPKD probands, 61 were male and 46 provided semen specimens for analysis. Specimens were collected by means of masturbation into a sterile container after $2-7$ days of abstinence. All specimens were assessed according to the World Health Organization (WHO) 2010 recommendations [9]. Briefly, within $1 \mathrm{~h}$ of ejaculation, the samples were liquefied and analysed for semen volume, sperm concentration, round cells, normal morphology, and sperm motility (defined as WHO motility grades A, B, C, and D, where grade A indicates fast progressive sperm; B, slow progressive sperm; C, nonprogressive sperm; and $\mathrm{D}$, immotile sperm).
All patients providing semen specimens for analysis had a normal 46, XY karyotype, and no Y chromosome abnormalities were detected by microdeletion detection. Other causes of infertility, such as drugs and exposure to toxic substances, were excluded. Physical examination of these male patients showed normal results, including height, weight, hair distribution, mental state, and external genital organs.

Most of individuals who provided semen specimens for analysis have chosen different approaches to conceive offspring, including natural pregnancy, in vitro fertilization (IVF), intracytoplasmic sperm injection (ICSI), and ICSI combined preimplantation genetic diagnosis (PGD).

\section{Mutation analysis of PKD1, PKD2, and GANAB}

Genomic DNA (gDNA) was extracted from peripheral blood samples using a QIAamp ${ }^{\circ}$ DNA blood midi kit (QIAGEN, Hilden, Germany) according to the manufacturer's protocol. All patients were subjected to mutation screening of PKD1 and PKD2 using Sanger sequencing, followed by multiplex ligation-dependent probe amplification analysis (MLPA) to detect copy number variation in PKD1 and PKD2 in patients lacking definitely pathogenic point mutations in PKD1 or PKD2. Subsequent screening of $G A N A B$ was carried out in patients for whom no causative genetic aetiology in PKD1 and PKD2 had been identified.

For exons 1-34 of PKD1, LR-PCR followed by nested PCR was performed with $P K D 1$-specific primers, as previously described [10-12], and exons 35-46 of PKD1 were directly amplified from gDNA by PCR. All exons of $P K D 2$ and GANAB, including the adjacent $30-60$ bp intron sequence, were amplified from gDNA by PCR. The primers for amplification of PKD1 were previously described, with minor modifications [12, 13]; specific primers for PKD2 and GANBA were designed using Primer 3 online (http://primer3.ut.ee) according to reference sequences. The primers and conditions for PCR reactions are provided in Additional file 1: Table S1, Additional file 2: Table S2, Additional file 3: Table S3, Additional file 4: Table S4. If a variant was identified as a putative disease-causative mutation, then mutation site screening of family members was implemented. DNA samples from all patients were screened by bidirectional sequencing on an Applied Biosystems 3130XL genetic analyser (Applied Biosystems, Foster City, CA, USA).

Copy number variation analysis of PKD1 and PKD2 was performed by MLPA with a SALSA MLPA probemix P351-C1/P352-D1 PKD1-PKD2 kit (MRC-Holland, Amsterdam, the Netherlands) according to the manufacturer's instructions [14]. This kit contains probes for 36 of the 46 exons of PKD1 and 17 probes for PKD2 exons, covering all PKD2 exons except exon 13 (two probes each for PKD2 exons 1, 2, and 6). The results of MLPA 
analysis were scanned on an Applied Biosystems 3130XL genetic analyser (Applied Biosystems, Foster City, CA, USA). The raw data were analysed using the Coffalyser MLPA analysis tool (MRC-Holland, Amsterdam, the Netherlands).

\section{Evaluation of the pathogenicity of variations}

The PKDB (http://pkdb.mayo.edu), the Human Gene Mutation Database (HGMD; http://www.hgmd.cf.ac.uk/ ac/index.php), Exome Aggregation Consortium (EXAC; http://exac.broadinstitute.org), and the gnomAD database (http://gnomad.broadinstitute.org) were searched for previously reported variations. Frameshift variations, typical splicing, nonsense, and in-frame changes of two or more amino acids were defined as definitely pathogenic mutations $[2,15]$. A novel mutation was defined as one that had not been described in PKDB or HGMD, or reported in ADPKD patients. The potential pathogenicity of all identified missense variants, indicated by a frequency below 1\% in the Asian population of the Exac and gnom $\mathrm{AD}$ databases, was evaluated by pedigree analysis and in silico analysis using three different tools: SIFT (http://sift.bii.a-star.edu.sg/), Polyphen-2 (http:// genetics.bwh.harvard.edu/pph2), and MutationTaster (http://www.mutationtaster.org/). All variants were classified into five categories: 'pathogenic', 'likely pathogenic', 'uncertain significance', 'likely benign', and 'benign', according to the American College of Medical Genetics and Genomics (ACMG) standards and guidelines for the interpretation of variations [16].

\section{Results}

\section{Mutation analysis of $P K D 1, P K D 2$, and GANAB}

We performed mutation screening of PKD1 and PKD2 for 90 probands using Sanger sequencing and MLPA. The 33 probands for whom no definitely pathogenic mutations were detected in PKD1 and PKD2 were subjected to screening of $G A N A B$. A total of 93 variations were identified in this study, comprising 84, 4, and 5 variations in $P K D 1, P K D 2$, and GANAB, respectively (Tables 1 and 2). Among these variations, 51 are novel and have not been described in the ADPKD Mutation Database or HGMD, or been reported in ADPKD patients.

\section{Evaluation of the pathogenicity of variations}

We evaluated the potential pathogenicity of all identified missense variants according to the ACMG standards and guidelines for the interpretation of variations. The results are shown in Tables 1 and 2. A total of 84 variations were identified in $P K D 1$, including 52 definitely pathogenic variations and 32 missense variants, 20 of which are classified as likely pathogenic mutations. Only four variants were identified in PKD2; three of them are definitely pathogenic mutations, and another is classified as likely to be pathogenic. Among the 76 definitely pathogenic or likely pathogenic mutations of $P K D 1$ and $P K D 2$, 38 are novel. We identified five novel variations in GANAB. Two variations (p.Arg173Gln, and p.Arg331Cys) have been reported in the gnomAD database 431, and 1979 times, respectively, including 3 , and 12 homozygotes, respectively, and are unlikely to be pathogenic. Three other variations (p.Pro123Ala, p.Met360Val, and p.Ile764Met) were identified in the gnomAD database 53,113 , and 44 times, respectively, and are not very highly conserved. Two of these (p.Pro123Ala and p.Ile764Met) and a likely pathogenic mutation in PKD1 (p.Leu727Pro) were identified in family 69 with co-occurrence, and segregated with the disease in three affected family members. However, since functional analysis has not been performed, we are unable to determine their pathogenicity thus far, and they are classified as variants of uncertain significance.

\section{Correlation with male infertility}

In order to analyze the correlation between ADPKD mutations and male infertility, we analyzed the types of PKD genes mutations and semen quality. In our study, the analysis of semen from 46 male patients revealed that sperm from 37 individuals were abnormal; asthenozoospermia was detected in 18 individuals; 18 other individuals were affected with oligozoospermia or oligoasthenozoospermia; and 1 individual suffered from azoospermia. A total of 28 of the individuals with abnormal sperm were found to harbour definitely pathogenic mutations, and 7 individuals with normal sperm also carried definitely pathogenic mutations (Table 3, Fig. 1). The results showed no correlation between semen quality and types of mutation in PKD genes.

A total of 35 patients who provided semen specimens for analysis have chosen different approaches to conceive. Two patients (one with asthenozoospermia and the other with oligoasthenozoospermia) conceived naturally, and two individuals (one with asthenozoospermia and the other with normal sperm) conceived through ICSI. The 31 other patients chose ICSI combined with PGD; of these patients, genetic diagnosis of embryos has been completed for 22 and the treatment cycle is currently incomplete for 9 patients (Table 4).

\section{Discussion}

In the present study, we analysed 90 unrelated patients affected with polycystic kidney disease, including 37 male patients with infertility. Screening of $P K D 1, P K D 2$, and $G A N A B$ was performed using a series of molecular genetic analyses. A total of 76 mutations (definitely or likely pathogenic mutations) were identified in 77 of the families, comprising 72 mutations in PKD1 and 4 in $P K D 2$. Pathogenic mutations in GANAB have never 
Table 1 Defnitely pathogenic mutations in PKD1 and PKD2 identified in this study

\begin{tabular}{|c|c|c|c|c|c|c|}
\hline cDNA change & Exon/ intron & Amino acid change & Mutation Type & Family No. & Family history & Known/Novel \\
\hline \multicolumn{7}{|l|}{ PKD1 } \\
\hline c.74dupG & 1 & p.Gly25Glyfs*89 & Frameshift & 29 & Yes & Novel \\
\hline c.106_107insT & 1 & p.Pro36Leufs*78 & Frameshift & 12 & Yes & Novel \\
\hline c.467_487del21 & 4 & p.Ala156_Ala162del & In-frame deletion & 49 & Yes & Novel \\
\hline c.856_862delTCTGGCC & 5 & p.Ser286Serfs*2 & Frameshift & 30 & Yes & Known \\
\hline c. $1198 \mathrm{C}>\mathrm{T}$ & 5 & p.Arg $400^{*}$ & Nonsense & 17 & Yes & Known \\
\hline c. 1297 C > T & 6 & p.G $\ln 433^{*}$ & Nonsense & 48 & NA & Known \\
\hline c. $2050 \mathrm{~A}>\mathrm{T}$ & 10 & p.Arg684* & Nonsense & 47 & Yes & Novel \\
\hline c.2659delT & 11 & p.Trp887Glyfs*11 & Frameshift & 50 & Yes & Known \\
\hline c. $2670+1 G>A$ & IVS14 & - & Splice & 19 & Yes & Novel \\
\hline c. $4177 \mathrm{C}>\mathrm{T}$ & 15 & p.Gln1393* & Nonsense & 51 & Yes & Novel \\
\hline \multirow[t]{2}{*}{ c. $.4447 C>T$} & 15 & p.Gln1483* & Nonsense & 13 & Yes & Known \\
\hline & & & & 14 & Yes & \\
\hline c. $4551 C>A$ & 15 & p.Tyr1517* & Nonsense & 39 & Yes & Novel \\
\hline c.4609G > T & 15 & p.Glu1537* & Nonsense & 31 & Yes & Known \\
\hline c.4846G $>\mathrm{T}$ & 15 & p.Glu1616* & Nonsense & 37 & Yes & Novel \\
\hline c. 4957 C > T & 15 & p.Gln1653* & Nonsense & 16 & Yes & Known \\
\hline c.5014_5015delAG & 15 & p.Arg1672Glyfs*98 & Frameshift & 53 & Yes & Known \\
\hline c.5120G > A & 15 & p.Trp1707* & Nonsense & 26 & Yes & Known \\
\hline c. $5637 C>G$ & 15 & p.Tyr1879* & Nonsense & 20 & Yes & Novel \\
\hline c. $6115 \mathrm{C}>\mathrm{T}$ & 15 & p.Gln2039* & Nonsense & 55 & Yes & Known \\
\hline c.6199C > T & 15 & p.Gln $2067^{*}$ & Nonsense & 34 & Yes & Known \\
\hline c.6804delG & 15 & p.Trp2268Cysfs*46 & Frameshift & 63 & No & Novel \\
\hline c.6813_6814delAC & 15 & p.Arg2272Glyfs*147 & Frameshift & 7 & Yes & Known \\
\hline c.6945_6946insT & 16 & p.Gly2316Trpfs*104 & Frameshift & 1 & Yes & Novel \\
\hline c.7126C $>\mathrm{T}$ & 17 & p.Gln $2376^{*}$ & Nonsense & 45 & Yes & Known \\
\hline c. $7863+1 G>C$ & IVS20 & - & Splice & 36 & Yes & Novel \\
\hline c.7863+2 T>G & IVS20 & - & Splice & 11 & Yes & Novel \\
\hline c.7915C > T & 21 & p.Arg2639* & Nonsense & 54 & Yes & Known \\
\hline c.7973_7974delTG & 21 & p.Val2658Glyfs*2 & Frameshift & 9 & Yes & Known \\
\hline c.8338G > T & 23 & p.Glu2780* & Nonsense & 27 & Yes & Known \\
\hline c.9666_9667delGA & 28 & p.Glu3222Aspfs*30 & Frameshift & 32 & Yes & Novel \\
\hline c. $10050+1 G>A$ & IVS30 & - & Splice & 44 & Yes & Known \\
\hline c. $10220+2 \mathrm{~T}>\mathrm{C}$ & IVS32 & - & Splice & 3 & Yes & Known \\
\hline c. 10397 C > G & 34 & p.Ser3466* & Nonsense & 6 & Yes & Novel \\
\hline c.10524_10525delAG & 35 & p.Glu3509Aspfs*117 & Frameshift & 2 & NA & Novel \\
\hline c.10710_10715delGGCTGT & 36 & p.3571_3572del2 & In-frame deletion & 40 & Yes & Known \\
\hline c. $10724 G>A$ & 36 & p.Try $3575^{*}$ & Nonsense & 38 & NA & Novel \\
\hline c.10896_10897delGA & 37 & p.Ser3633Profs*88 & Frameshift & 5 & No & Novel \\
\hline c.11240delC & 39 & p.Pro3747Hisfs*79 & Frameshift & 33 & Yes & Novel \\
\hline c. $11269+1 G>A$ & IVS39 & - & Splice & 10 & Yes & Novel \\
\hline c.11311_11312insGTGCT & 40 & p.Ser3771Cysfs*57 & Frameshift & 41 & NA & Novel \\
\hline c. $11512 C>T$ & 41 & p.Gln3838* & Nonsense & 15 & Yes & Known \\
\hline c. $11538-2 A>G$ & IVS41 & - & Splice & 18 & Yes & Known \\
\hline
\end{tabular}


Table 1 Defnitely pathogenic mutations in PKD1 and PKD2 identified in this study (Continued)

\begin{tabular}{|c|c|c|c|c|c|c|}
\hline cDNA change & Exon/ intron & Amino acid change & Mutation Type & Family No. & Family history & Known/Novel \\
\hline c.11617_11637del21 & 42 & p.3873_3879del7 & In-frame deletion & 4 & Yes & Novel \\
\hline c.11699_11700ins10 & 42 & p.Leu3901Alafs*63 & Frameshift & 22 & Yes & Novel \\
\hline c.11830_11838dup & 43 & p.Leu3944_Ala3946dup & In-frame duplication & 52 & Yes & Novel \\
\hline c.12101delT & 44 & p.Val4034Glyfs*5 & Frameshift & 25 & No & Novel \\
\hline c. $12139-2 A>T$ & IVS44 & - & Splice & 24 & Yes & Novel \\
\hline c.12391G > T & 45 & p.Glu4131* & Nonsense & 43 & Yes & Known \\
\hline c.12570_12571insCTCC & 46 & p.Ser4190Serfs*21 & Frameshift & 28 & Yes & Novel \\
\hline \multirow[t]{2}{*}{ c. $12682 C>T$} & 46 & p.Arg4228* & Nonsense & 21 & Yes & Known \\
\hline & & & & 23 & Yes & \\
\hline c. $12712 C>T$ & 46 & p.Gln4238* & Nonsense & 46 & Yes & Known \\
\hline EX31-33del & $31-33$ & - & Large deletion & 72 & No & Novel \\
\hline \multicolumn{7}{|l|}{ PKD2 } \\
\hline c. $973 \mathrm{C}>\mathrm{T}$ & 4 & p.Arg325* & Nonsense & 8 & Yes & Known \\
\hline c.1094 + 3_1094 + 6delAAGT & IVS4 & - & Splice & 35 & Yes & Known \\
\hline c.2159dupA & 11 & p.Asn720Lysfs*5 & Frameshift & 42 & Yes & Known \\
\hline
\end{tabular}

NA not available; *translation termination codon. Novel mutation defined as one that had not been described in PKDB, HGMD, or reported in ADPKD patients

been identified in Chinese patients. To our knowledge, this is the first report of GANAB screening in a cohort of Chinese patients with ADPKD.

$P K D 1, P K D 2$, and GANAB are located in chromosome regions $16 \mathrm{p} 13.3,4 \mathrm{q} 21-22$, and $11 \mathrm{q} 12.3$, and they produce the proteins polycystin-1 (PC-1), PC-2, and neutral alpha-glucosidase $A B$, respectively [17]. A series of molecular genetic analyses were used to screen for mutations of PKD genes. The human genome contains six truncated PKD1 pseudogenes, which share approximately $97.7 \%$ similarity with exons $1-34$ of PKD1 [18]. PKD1 contains complex reiterated regions, necessitating that LR-PCR be performed prior to sequencing [19]. Screening of PKD2 was performed by direct Sanger sequencing. Subsequently, MLPA was employed to analyse the copy number variations of $P K D 1$ and $P K D 2$ in the genetically unresolved families, followed by screening of $G A N A B$ using direct Sanger sequencing. This strategy can typically identify almost all variants in PKD genes, as verified by our high mutation detection rate $(85.6 \%, 77 / 90)$. Recently, targeted next-generation sequencing and whole-exome sequencing have been used to identify mutations involved in ADPKD. Although these methods have high sensitivity, specificity, and accuracy, LR-PCR is still required; furthermore, highly specialised personnel and expensive equipment are required [20]. Thus, the strategy for the identification of mutations in ADPKD used in the present study may be useful in a wide variety of situations.

A total of 2609 variants had been described before January 2018 (2323 in PKD1, 278 in PKD2, and 8 in $G A N A B)$. The majority of these variants were missense variants (1225). The others were protein-truncating variants (840), splice site mutations (165), in-frame indels (115), large deletions (24), and variations in the UTR and intervening sequences (228). In our study, a total of 76 mutations (definitely or likely pathogenic variations) were identified in 77 of the families, comprising 41 protein-truncating, 21 missense mutations, and 9 splice site mutations; 4 in-frame indels; and 1 large deletion variants. The positive detection rate was $85.6 \%$ (77/ $90)$, and $50 \%(38 / 76)$ of all mutations were novel. The proportion of patients with ADPKD with a family history of the disease accounted for $80 \%$ (72/90) of all probands, comparable to previously published data [21].

A total of 76 mutations were identified, 72 of which (including 52 definitely pathogenic mutations) were in PKD1, accounting for $94.7 \%$. Among all definitely pathogenic mutations of PKD1, 39 were truncating mutations, accounting for a large proportion $(75 \%, 39 / 52)$, concordant with the results of other recent studies [17]. Furthermore, one large deletion of PKD1 was identified in our set of patients $(1.1 \%, 1 / 90)$, in accordance with previously reported results [22, 23]. A total of 4 mutations were identified in PKD2, 3 of which were definitely pathogenic mutations. However, no hot-spots of mutation were identified in $P K D 1$ or $P K D 2$, indicating that for identification of future mutations, all exons of $P K D 1$ and $P K D 2$, including their intron-exon boundaries, should be sequenced.

$G A N A B$ has been implicated in the development of autosomal-dominant polycystic kidney and liver disease [7]. In this study, a total of five mutations with a frequency below $1 \%$ were identified in the Asian population of the Exac and gnomAD databases; all are missense 


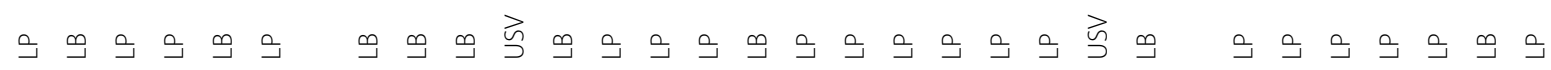

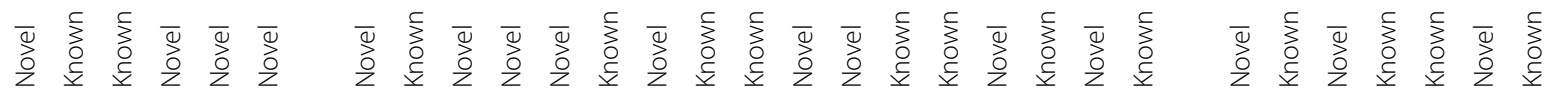

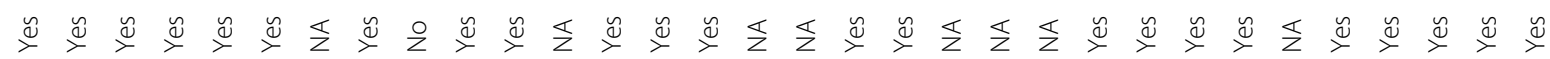

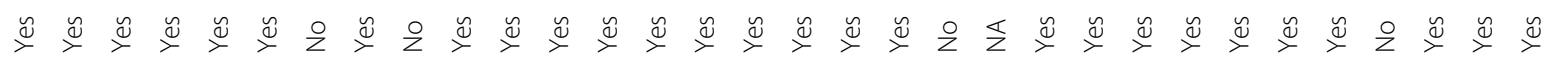

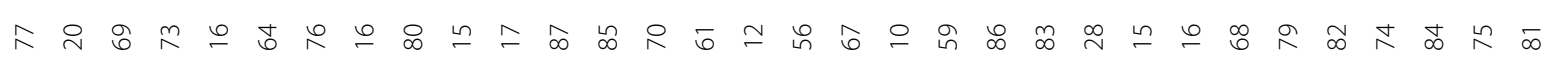

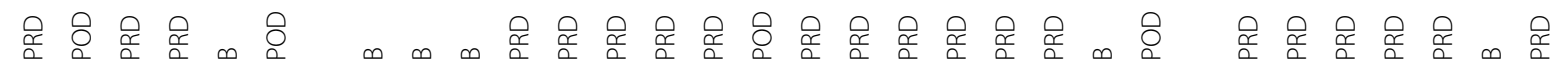

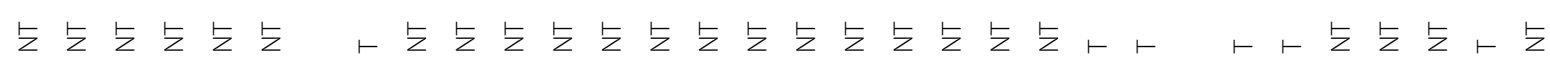

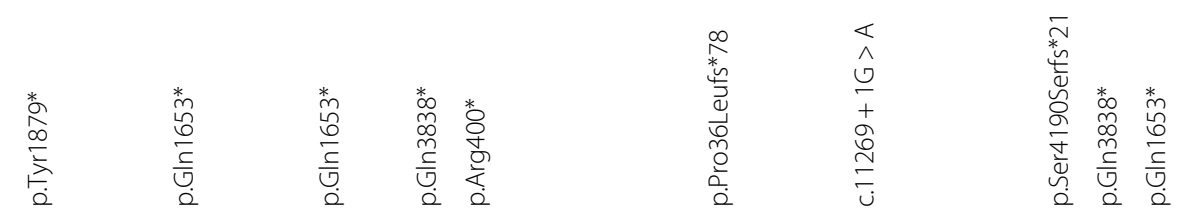

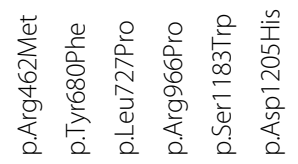

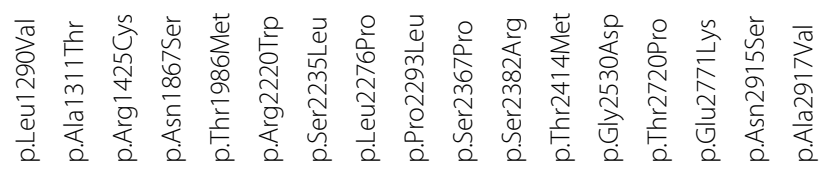

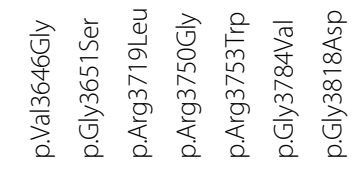

$0 \odot=\simeq \stackrel{\operatorname{Ln}}{\leftarrow}$

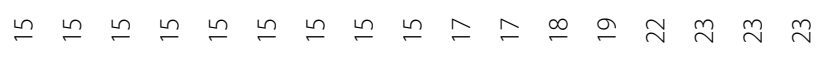

$\hat{m} \hat{m} \stackrel{\infty}{m} \stackrel{\dot{m}}{\dot{m}}$ q $ซ$

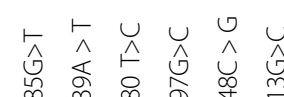

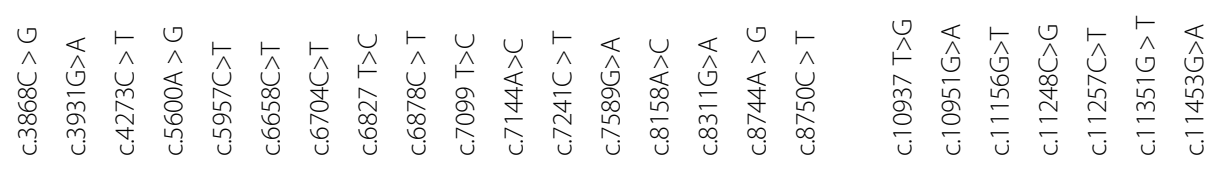




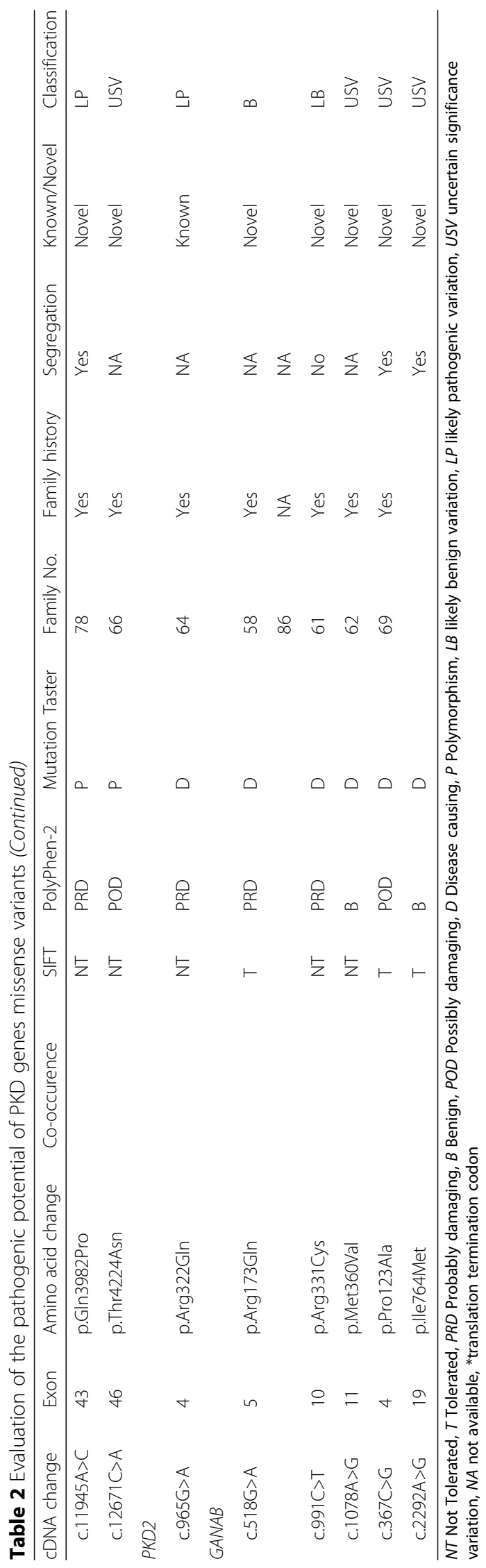




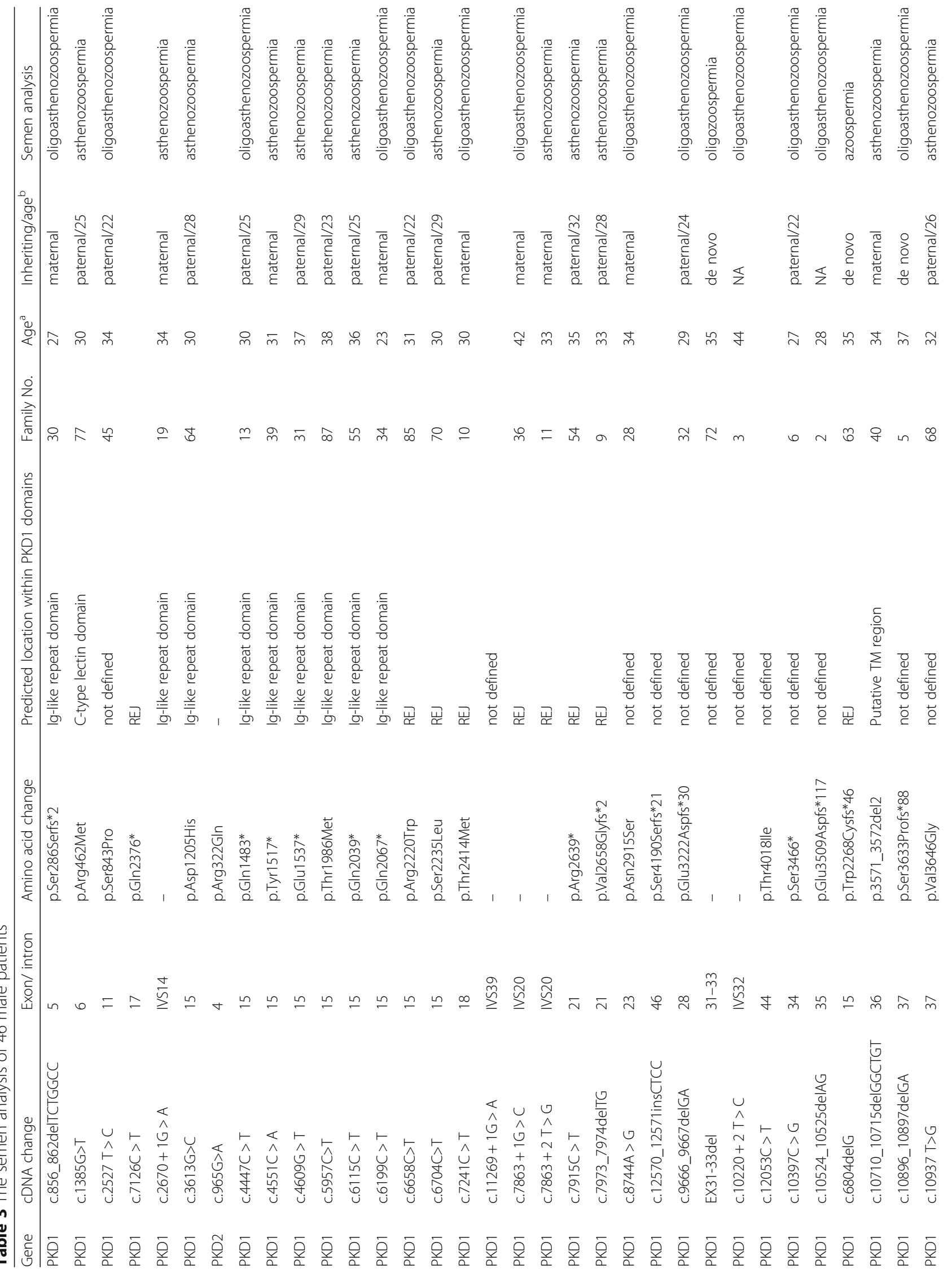




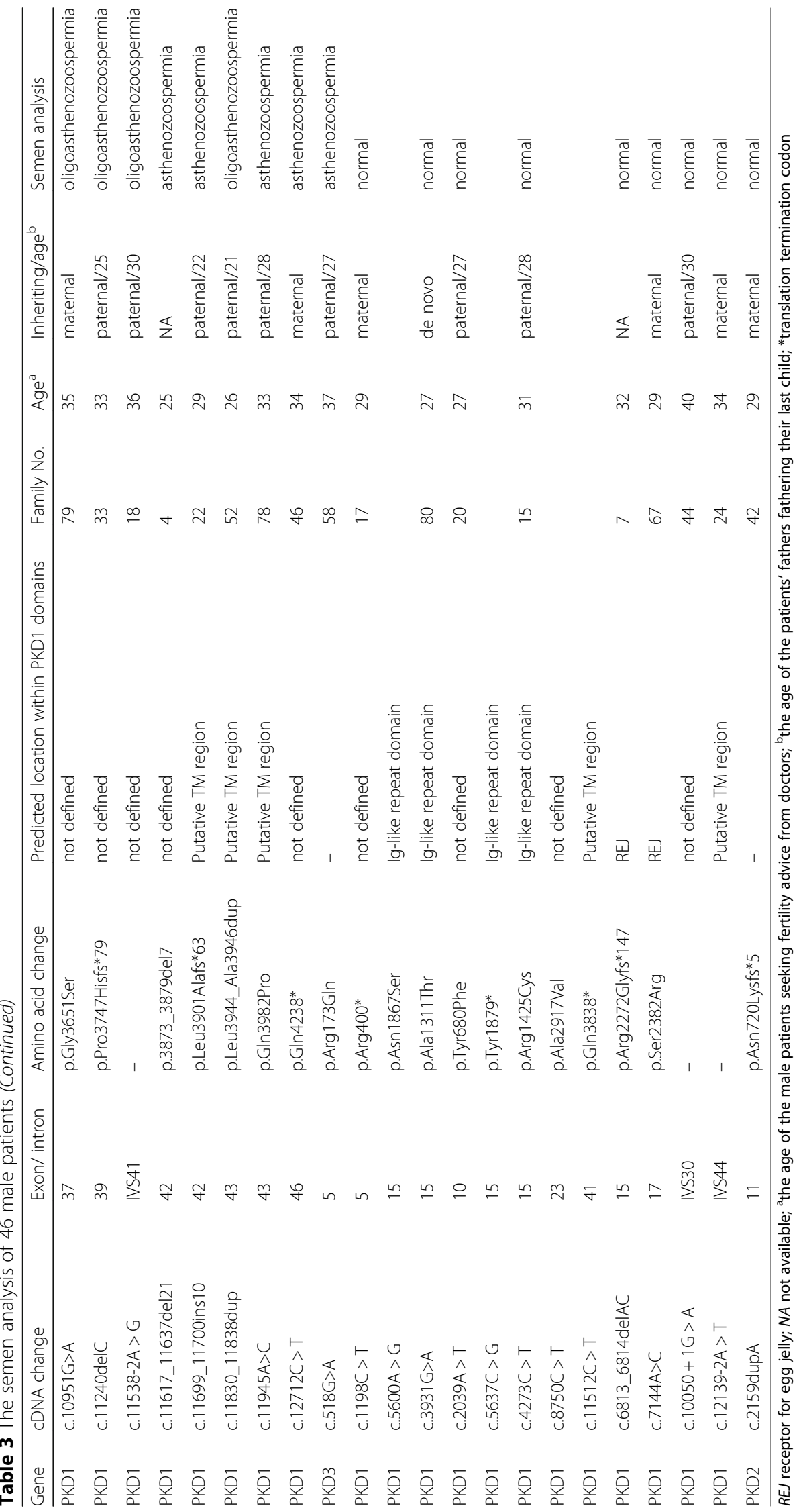




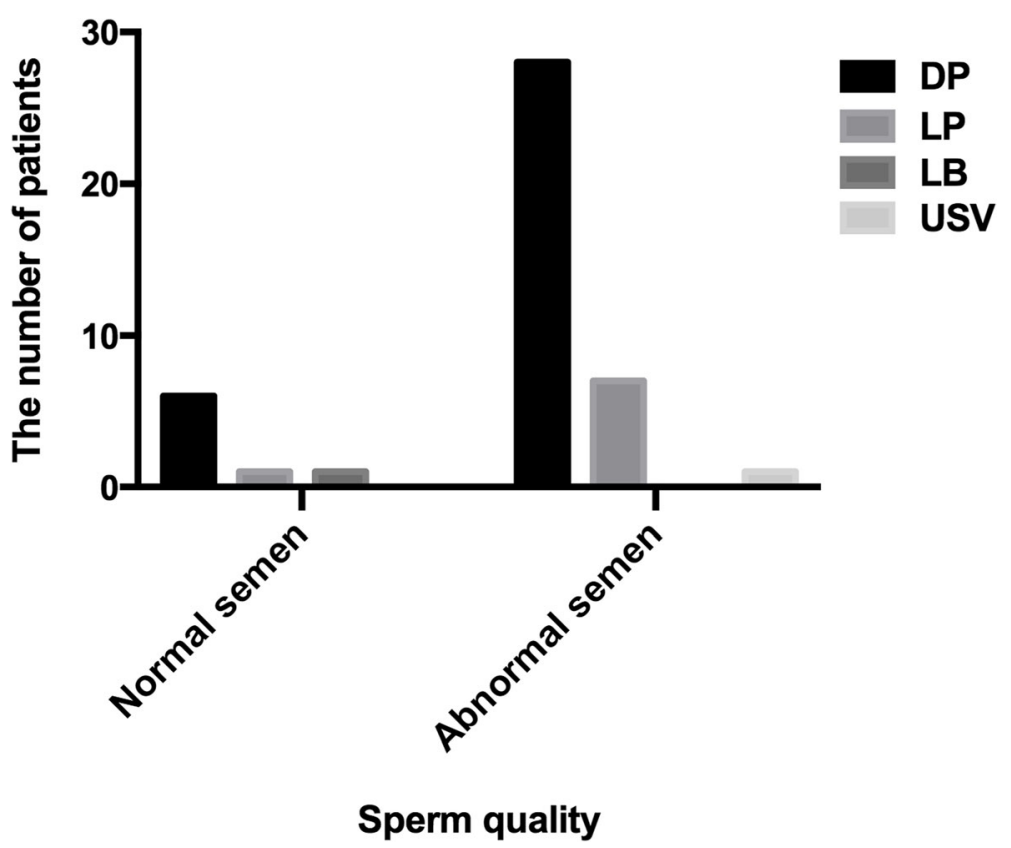

Fig. 1 The semen quality of the male patients who harboured PKD1 mutations. DP, LP, LB and USV are indicated with definitely pathogenic mutations, likely pathogenic variations, likely benign variations and uncertain significance variations, respectively. The results showed that there is no correlation between semen quality and the type of mutation in PKD1 gene

mutations. Only two mutations (p.Pro123Ala and p.Ile764Met) co-occurred in family 69; these have been described a few times and were found to have segregated with the disease in affected family members, accounting for $1.1 \%$. The patients in this family all suffered from polycystic kidney with liver disease, consistent with earlier findings that the phenotype caused by GANAB mutations usually manifests with polycystic liver disease (PLD) [7]. In addition, the patients from family 69 also carried the p.Leu727Pro mutation in PKD1, which has been reported in several families and classified as a highly likely pathogenic mutation [17, 24]. As p.Pro123Ala was predicted to be a disease-causing mutation by three tools and p.Ile764Met was predicted to be benign by PolyPhen-2, the possibility that p.Leu727Pro in PKD1 and p.Pro123Ala in $G A N A B$ co-contribute to the development of polycystic kidney with liver disease cannot be excluded. Thus, the fact that no definitely or likely pathogenic mutation was detected in this study suggested that GANAB mutations are rare in Chinese patients with ADPKD.

Earlier studies have reported that $H N F 1 B$ can phenocopy ADPKD [25-27]. In addition, it has been very recently reported that monoallelic mutation in DNAJB11 can cause atypical ADPKD, which is a phenotypic hybrid of ADPKD and autosomal-dominant tubulointerstitial diseases (ADTKD) [28]. In our study, for the 13 patients with or without a positive family history, the genetic cause remains unknown, but undetected PKD1, PKD2, or GANAB mutations, including deep intronic or synonymous exonic mutations that cause atypical splicing, or large deletions of $G A N A B$, could be the underlying reasons. Furthermore, the patients should be reevaluated based on their most recent phenotype, and should be screened for other genes implicated in ADPKD for future analysis, such as $H N F 1 B$ and DNAJB11.

ADPKD is a systemic disorder and extrarenal manifestation is not uncommon. Male patients with ADPKD usually suffer from infertility, resulting from abnormal semen, including necrospermia, immotile sperm, asthenozoospermia, and azoospermia [29-31]. PKD1 and PKD2 have been reported to play a pivotal role in the development and maintenance of the male reproductive tract [32, 33]. The potential aetiologies of semen abnormalities in male patients with ADPKD include ejaculatory duct cysts, seminal vesicle cysts, and ultrastructural flagellar defects caused by abnormal polycystins [30]. However, the correlation between the type of PKD gene mutation and semen quality remains unclear. In the present study, 37 individuals were found to have abnormal semen $(80 \%, 37 / 46)$. Only some of the male patients with ADPKD carrying definitely pathogenic mutations were infertile, which may indicate that there is no correlation between the type of PKD1 mutation and semen quality. However, 23 of the variations are located in the same four domains of PKD1, and more than one third of the mutations $(39 \%, 9 / 23)$ are located in the Ig-like repeat domain, which is a conserved region of approximately 85 bp surrounding a central sequence consisting of 16 copies [34]. Defects of the Ig-like 
Table 4 The assisted reproductive therapies used by the 35 male patients and the clinical outcomes of those therapies

\begin{tabular}{|c|c|c|c|c|c|}
\hline Gene & cDNA change & Amino acid change & Family No. & Semen analysis & Treatment methods \\
\hline PKD1 & c.856_862delTCTGGCC & p.Ser286Serfs 2 & 30 & oligoasthenozoospermia & ICSI+PGD \\
\hline PKD1 & c.1385G>T & p.Arg462Met & 77 & asthenozoospermia & $|C S|+P G D$ \\
\hline PKD1 & c. $2527 \mathrm{~T}>\mathrm{C}$ & p.Ser843Pro & 45 & oligoasthenozoospermia & ICSI+PGD \\
\hline PKD1 & c. $7126 C>T$ & p.G $\ln 2376^{*}$ & & & \\
\hline PKD1 & c. $2670+1 G>A$ & - & 19 & asthenozoospermia & $|C S|+P G D^{a}$ \\
\hline PKD1 & c. $4447 \mathrm{C}>\mathrm{T}$ & p.G $\ln 1483^{*}$ & 13 & oligoasthenozoospermia & $|C S|+P G D^{a}$ \\
\hline PKD1 & c. $4551 C>A$ & p.Tyr $1517^{*}$ & 39 & asthenozoospermia & natural pregnant \\
\hline PKD1 & c. $4609 \mathrm{G}>\mathrm{T}$ & p.Glu1537* & 31 & asthenozoospermia & $|C S|+P G D$ \\
\hline PKD1 & c. $6199 C>T$ & p.Gln2067 & 34 & oligoasthenozoospermia & natural pregnant \\
\hline PKD1 & c.6658C $>\mathrm{T}$ & p.Arg2220Trp & 85 & oligoasthenozoospermia & $I C S I+P G D$ \\
\hline PKD1 & c.6704C>T & p.Ser2235Leu & 70 & asthenozoospermia & $|C S|+P G D$ \\
\hline PKD1 & c. $7863+1 G>C$ & - & 36 & oligoasthenozoospermia & $|C S|+P G D$ \\
\hline PKD1 & $c .7863+2 \mathrm{~T}>\mathrm{G}$ & - & 11 & asthenozoospermia & $|C S|+P G D$ \\
\hline PKD1 & c. $10529 C>T$ & p.Thr3510Met & & & \\
\hline PKD1 & c.7915C > T & p.Arg $2639^{*}$ & 54 & asthenozoospermia & $|C S|+P G D$ \\
\hline PKD1 & c.7973_7974delTG & p.Val2658Glyfs ${ }^{*} 2$ & 9 & asthenozoospermia & ICSI \\
\hline PKD1 & c. $10529 C>T$ & p.Thr3510Met & & & \\
\hline PKD1 & c. $8744 A>G$ & p.Asn2915Ser & 28 & oligoasthenozoospermia & $|C S|+P G D^{a}$ \\
\hline PKD1 & c.12570_12571insCTCC & p.Ser4190Serfs 21 & & & \\
\hline PKD1 & c.9666_9667delGA & p.Glu3222Aspfs ${ }^{*} 30$ & 32 & oligoasthenozoospermia & $|C S|+P G D^{a}$ \\
\hline PKD1 & c. $10220+2 \mathrm{~T}>\mathrm{C}$ & - & 3 & oligoasthenozoospermia & $|C S|+P G D^{a}$ \\
\hline PKD1 & c. $12053 C>T$ & p.Thr4018lle & & & \\
\hline PKD1 & c.10524_10525delAG & p.Glu3509Aspfs*117 & 2 & oligoasthenozoospermia & $|C S|+P G D$ \\
\hline PKD1 & c.10896_10897delGA & p.Ser3633Profs*88 & 5 & oligoasthenozoospermia & $\mid C S I+P G D^{a}$ \\
\hline PKD1 & c. $10937 \mathrm{~T}>\mathrm{G}$ & p.Val3646Gly & 68 & asthenozoospermia & $|C S|+P G D$ \\
\hline PKD1 & c.11240delC & p.Pro3747Hisfs 79 & 33 & oligoasthenozoospermia & $|C S|+P G D^{a}$ \\
\hline PKD1 & c. $11538-2 A>G$ & - & 18 & oligoasthenozoospermia & $|C S|+P G D$ \\
\hline PKD1 & c.11699_11700ins10 & p.Leu3901Alafs" 63 & 22 & asthenozoospermia & $|C S|+P G D$ \\
\hline PKD1 & c.11830_11838dup & p.Leu3944_Ala3946dup & 52 & oligoasthenozoospermia & $|C S|+P G D$ \\
\hline PKD1 & C. $11945 A>C$ & p.Gln3982Pro & 78 & asthenozoospermia & $|C S|+P G D$ \\
\hline PKD1 & c. $12712 C>T$ & p.Gln4238* & 46 & asthenozoospermia & $|C S|+P G D$ \\
\hline PKD1 & c. $1198 C>T$ & p.Arg $400^{*}$ & 17 & normal & ICSI+PGD \\
\hline PKD1 & C. $5600 A>G$ & p.Asn1867Ser & & & \\
\hline PKD1 & c.3931G >A & p.Ala1311Thr & 80 & normal & $|\mathrm{ICS}|$ \\
\hline PKD1 & c. $2039 \mathrm{~A}>\mathrm{T}$ & p.Tyr680Phe & 20 & normal & $|C S|+P G D^{a}$ \\
\hline PKD1 & c. $5637 C>G$ & p.Tyr $1879^{*}$ & & & \\
\hline PKD1 & $c .4273 C>T$ & p.Arg1425Cys & 15 & normal & $|C S|+P G D^{a}$ \\
\hline PKD1 & $c .8750 C>T$ & p.Ala2917Val & & & \\
\hline PKD1 & c. $11512 C>T$ & p.G $\ln 3838^{*}$ & & & \\
\hline PKD1 & c.6813_6814delAC & p.Arg2272Glyfs ${ }^{*} 147$ & 7 & normal & $\mid \mathrm{CSI}+\mathrm{PGD}$ \\
\hline PKD1 & C. $7144 A>C$ & p.Ser2382Arg & 67 & normal & $\mid \mathrm{CSI}+\mathrm{PGD}$ \\
\hline PKD1 & $c .10050+1 G>A$ & - & 44 & normal & $|C S|+P G D$ \\
\hline PKD1 & c. $12139-2 A>T$ & - & 24 & normal & $\mid \mathrm{CSI}+\mathrm{PGD}$ \\
\hline PKD2 & c.2159dupA & p.Asn720Lysfs ${ }^{*} 5$ & 42 & normal & ICSI+PGD \\
\hline
\end{tabular}

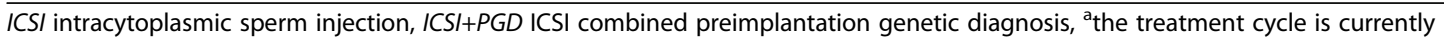
incomplete, *translation termination codon 
repeat domain in PKD1 protein may alter its binding ability, leading to male reproductive tract cysts and infertility [34]. Thus, male patients carrying pathogenic mutations in PKD1 located in the Ig-like repeat domain may have a high risk of infertility. In addition, 20 and 3 of the mutations identified in the 23 male patients with ADPKD with abnormal semen were paternal and de novo, respectively. However, almost all of these fathers fathered their children when they were younger than the age at which their sons with ADPKD sought fertility advice from their doctors. Furthermore, after the treatment of ICSI, 17 individuals affected with semen abnormalities all achieved paternity or at least obtained embryos. Therefore, we suggest that male patients with ADPKD should achieve paternity as young as possible, and the use of ICSI combined PGD should be considered for patients suffering from low semen quality [30, 31].

In this study, the majority of male patients with ADPKD were found to have abnormal semen (80\%), which could due to a selection bias in the study population. Since our hospital specializes in reproductive and genetic disorders, most of the subjects included in the study visited our hospital to seek treatment for infertility. Thus, the proportion of males with abnormal sperm quality is not a true reflection of the proportion of male ADPKD patients with abnormal sperm. Therefore, studies on larger groups of patients with ADPKD recruited from general hospitals are needed to obtain a more accurate estimation of the proportion of ADPKD-affected males with abnormal sperm.

\section{Conclusions}

In conclusion, we identified a group of novel mutations in PKD genes, which enriches the PKD mutation spectrum. Male patients with ADPKD are usually affected with infertility, and surgical sperm retrieval combined with assisted reproductive technology may help them to achieve paternity. Our study will provide clinicians with precise diagnoses that have implications for family planning and genetic counselling of affected individuals.

\section{Additional files}

Additional file 1: Table S1. Primers used in long-range PCR of PKD1

homologous regions. (DOC $37 \mathrm{~kb}$ )

Additional file 2: Table S2. Primers used in nested PCR of PKD1 homologous regions. (DOC $78 \mathrm{~kb}$ )

Additional file 3: Table S3. Primers used in PCR of PKD1 single-copy regions. (DOC $40 \mathrm{~kb}$ )

Additional file 4: Table S4. Primers used in PCR of PKD2 and GANAB. (DOC $53 \mathrm{~kb}$ )

\section{Abbreviations}

ACMG: American College of Medical Genetics and Genomics:

ADPKD: Autosomal dominant polycystic kidney disease; ESRD: End-stage renal disease; HGMD: Human gene mutation database; ICSI: Intracytoplasmic sperm injection; LR-PCR: Long-range PCR; MLPA: Multiplex ligationdependent probe amplification; PC-1: Produce the proteins polycystin-1; PC2: Produce the proteins polycystin-2; PKDB: Autosomal dominant polycystic kidney disease mutation database; PLD: Polycystic liver disease; WHO: World Health Organization

\section{Acknowledgements}

We would like to thank the patients and their family members for their support and participation in this research. We would also like to thank the genetic counselling team at the Reproductive and Genetic Hospital of CITICXiangya and the clinicians who referred the patients for the clinical study.

\section{Author' contributions}

JD designed the study. WBH, WJX, XMZ, QJZ, and WL performed the mutation analysis of PKD1, PKD2, and GANAB. JD and WBH carried out the evaluation of the pathogenicity of variations. CGZ, XRL, LH, GXL, and GL worked on the clinical study. WBH, WJX and YQT wrote the paper. All authors read and approved the final manuscript.

\section{Funding}

This study was supported by grants from the National Natural Science Foundation of China (81771645 and 81471432), Scientific Research Foundation of Reproductive and Genetic Hospital of CITIC-Xiangya (YNXM201802) and Graduate Research and Innovation Projects of Central South University (Grant 2017zzts372).

\section{Availability of data and materials}

The datasets used and/or analysed during the current study available from the corresponding author on reasonable request.

\section{Ethics approval and consent to participate}

Approval of this study was obtained from Reproductive and Genetic Hospital of CITIC-Xiangya. Written informed consent for analyses was obtained from all participants.

\section{Consent for publication}

No individual patient data has been reported in the current study and consent for publication is not applicable.

\section{Competing interests}

The authors declare that they have no competing interests.

\section{Publisher's Note}

Springer Nature remains neutral with regard to jurisdictional claims in published maps and institutional affiliations.

Received: 5 March 2018 Accepted: 24 September 2018

Published online: 17 October 2018

\section{References}

1. Ong AC, Devuyst O, Knebelmann B, Walz G, Diseases E-EWGIK. Autosomal dominant polycystic kidney disease: the changing face of clinical management. Lancet. 2015;385(9981):1993-2002.

2. Rossetti S, Consugar MB, Chapman AB, Torres VE, Guay-Woodford LM, Grantham JJ, et al. Comprehensive molecular diagnostics in autosomal dominant polycystic kidney disease. J Am Soc Nephrol. 2007;18(7):2143-60.

3. Mieusset R, Fauquet I, Chauveau D, Monteil L, Chassaing N, Daudin M, et al. The spectrum of renal involvement in male patients with infertility related to excretory-system abnormalities: phenotypes, genotypes, and genetic counseling. J Nephrol. 2017;30(2):211-8.

4. European Polycystic Kidney Disease Consortium. The polycystic kidney disease 1 gene encodes a $14 \mathrm{~kb}$ transcript and lies within a duplicated region on chromosome 16. The European Polycystic Kidney Disease Consortium. Cell. 1994;77(6):881-94.

5. Kimberling WJ, Kumar S, Gabow PA, Kenyon JB, Connolly CJ, Somlo S. Autosomal dominant polycystic kidney disease: localization of the second gene to chromosome 4q13-q23. Genomics. 1993;18(3):467-72.

6. Iliuta IA, Kalatharan V, Wang K, Cornec-Le Gall E, Conklin J, Pourafkari M, et al. Polycystic kidney disease without an apparent family history. J Am Soc Nephrol. 2017;28(9):2768-76. 
7. Porath B, Gainullin VG, Cornec-Le Gall E, Dillinger EK, Heyer CM, Hopp K, et al. Mutations in GANAB, encoding the glucosidase llalpha subunit, cause autosomal-dominant polycystic kidney and liver disease. Am J Hum Genet. 2016;98(6):1193-207.

8. Pei Y, Obaji J, Dupuis A, Paterson AD, Magistroni R, Dicks E, et al. Unified criteria for ultrasonographic diagnosis of ADPKD. J Am Soc Nephrol. 2009; 20(1):205-12.

9. Cooper TG, Noonan E, von Eckardstein S, Auger J, Baker HW, Behre HM, et al. World Health Organization reference values for human semen characteristics. Hum Reprod Update. 2010;16(3):231-45.

10. Tan YC, Blumenfeld JD, Anghel R, Donahue S, Belenkaya R, Balina M, et al. Novel method for genomic analysis of PKD1 and PKD2 mutations in autosomal dominant polycystic kidney disease. Hum Mutat. 2009;30(2):264-73.

11. Phakdeekitcharoen B, Watnick TJ, Germino GG. Mutation analysis of the entire replicated portion of PKD1 using genomic DNA samples. J Am Soc Nephrol. 2001;12(5):955-63

12. Zhang S, Mei C, Zhang D, Dai B, Tang B, Sun T, et al. Mutation analysis of autosomal dominant polycystic kidney disease genes in Han Chinese. Nephron Exp Nephrol. 2005;100(2):e63-76.

13. Rossetti S, Chauveau D, Walker D, Saggar-Malik A, Winearls CG, Torres VE, et al. A complete mutation screen of the ADPKD genes by DHPLC. Kidney Int. 2002;61(5):1588-99.

14. Schouten JP, McElgunn CJ, Waaijer R, Zwijnenburg D, Diepvens F, Pals G. Relative quantification of 40 nucleic acid sequences by multiplex ligationdependent probe amplification. Nucleic Acids Res. 2002;30(12):e57.

15. Liu B, Chen SC, Yang YM, Yan K, Qian YQ, Zhang JY, et al. Identification of novel PKD1 and PKD2 mutations in a Chinese population with autosomal dominant polycystic kidney disease. Sci Rep. 2015;5:17468.

16. Richards S, Aziz N, Bale S, Bick D, Das S, Gastier-Foster J, et al. Standards and guidelines for the interpretation of sequence variants: a joint consensus recommendation of the American College of Medical Genetics and Genomics and the Association for Molecular Pathology. Genet Med. 2015; 17(5):405-24.

17. Cornec-Le Gall E, Audrezet MP, Chen JM, Hourmant M, Morin MP, Perrichot $R$, et al. Type of PKD1 mutation influences renal outcome in ADPKD. J Am Soc Nephrol. 2013;24(6):1006-13.

18. Symmons O, Varadi A, Aranyi T. How segmental duplications shape our genome: recent evolution of ABCC6 and PKD1 Mendelian disease genes. Mol Biol Evol. 2008;25(12):2601-13.

19. Rossetti S, Strmecki L, Gamble V, Burton S, Sneddon V, Peral B, et al. Mutation analysis of the entire PKD1 gene: genetic and diagnostic implications. Am J Hum Genet. 2001;68(1):46-63.

20. Qi XP, Du ZF, Ma JM, Chen XL, Zhang Q, Fei J, et al. Genetic diagnosis of autosomal dominant polycystic kidney disease by targeted capture and nextgeneration sequencing: utility and limitations. Gene. 2013;516(1):93-100.

21. Neumann HP, Bacher J, Nabulsi Z, Ortiz Bruchle N, Hoffmann MM, Schaeffner E, et al. Adult patients with sporadic polycystic kidney disease: the importance of screening for mutations in the PKD1 and PKD2 genes. Int Urol Nephrol. 2012;44(6):1753-62.

22. Obeidova L, Elisakova V, Stekrova J, Reiterova J, Merta M, Tesar V, et al. Novel mutations of PKD genes in the Czech population with autosomal dominant polycystic kidney disease. BMC Med Genet. 2014;15:41.

23. Consugar MB, Wong WC, Lundquist PA, Rossetti S, Kubly VJ, Walker DL, et al. Characterization of large rearrangements in autosomal dominant polycystic kidney disease and the PKD1/TSC2 contiguous gene syndrome. Kidney Int. 2008;74(11):1468-79.

24. Rossetti S, Hopp K, Sikkink RA, Sundsbak JL, Lee YK, Kubly V, et al. Identification of gene mutations in autosomal dominant polycystic kidney disease through targeted resequencing. J Am Soc Nephrol. 2012;23(5):915-33.

25. Verhave JC, Bech AP, Wetzels JF, Nijenhuis T. Hepatocyte nuclear factor 1 beta-associated kidney disease: more than renal cysts and diabetes. J Am Soc Nephrol. 2016;27(2):345-53.

26. Nishigori H, Yamada S, Kohama T, Tomura H, Sho K, Horikawa Y, et al. Frameshift mutation, A263fsinsGG, in the hepatocyte nuclear factor-1beta gene associated with diabetes and renal dysfunction. Diabetes. 1998;47(8):1354-5.

27. Pace NP, Craus J, Felice A, Vassallo J. Case report: identification of an HNF1B p.Arg527GIn mutation in a Maltese patient with atypical early onset diabetes and diabetic nephropathy. BMC Endocr Disord. 2018;18(1):28

28. Cornec-Le Gall E, Olson RJ, Besse W, Heyer CM, Gainullin VG, Smith JM, et al. Monoallelic mutations to DNAJB11 cause atypical autosomal-dominant polycystic kidney disease. Am J Hum Genet. 2018;102(5):832-44.
29. Torra R, Sarquella J, Calabia J, Marti J, Ars E, Fernandez-Llama P, et al. Prevalence of cysts in seminal tract and abnormal semen parameters in patients with autosomal dominant polycystic kidney disease. Clin J Am Soc Nephrol. 2008;3(3):790-3.

30. Kim JA, Blumenfeld JD, Prince MR. Seminal vesicles in autosomal dominant polycystic kidney disease. In: Li X, editor. Polycystic kidney disease. Brisbane (AU); 2015.

31. Shefi S, Levron J, Nadu A, Raviv G. Male infertility associated with adult dominant polycystic kidney disease: a case series. Arch Gynecol Obstet. 2009;280(3):457-60.

32. Nie $X$, Arend LJ. Pkd1 is required for male reproductive tract development. Mech Dev. 2013;130(11-12):567-76

33. Nie $\mathrm{X}$, Arend LJ. Novel roles of $\mathrm{Pkd} 2$ in male reproductive system development. Differentiation. 2014;87(3-4):161-71.

34. Hughes J, Ward CJ, Peral B, Aspinwall R, Clark K, San Millan JL, et al. The polycystic kidney disease 1 (PKD1) gene encodes a novel protein with multiple cell recognition domains. Nat Genet. 1995;10(2):151-60.
Ready to submit your research? Choose BMC and benefit from:

- fast, convenient online submission

- thorough peer review by experienced researchers in your field

- rapid publication on acceptance

- support for research data, including large and complex data types

- gold Open Access which fosters wider collaboration and increased citations

- maximum visibility for your research: over $100 \mathrm{M}$ website views per year

At BMC, research is always in progress.

Learn more biomedcentral.com/submissions 